\title{
Realization of Microwave Quantum Circuits Using Hybrid Superconducting-Semiconducting Nanowire Josephson Elements
}

\author{
G. de Lange, ${ }^{1}$ B. van Heck, ${ }^{2}$ A. Bruno, ${ }^{1}$ D. J. van Woerkom, ${ }^{1}$ A. Geresdi, ${ }^{1}$ S. R. Plissard,${ }^{3}$ \\ E. P. A. M. Bakkers, ${ }^{1,3}$ A. R. Akhmerov, ${ }^{1}$ and L. DiCarlo ${ }^{1}$ \\ ${ }^{1}$ QuTech and Kavli Institute of Nanoscience, Delft University of Technology, 2600 GA Delft, The Netherlands \\ ${ }^{2}$ Instituut-Lorentz, Leiden University, 2300 RA Leiden, The Netherlands \\ ${ }^{3}$ Department of Applied Physics, Eindhoven University of Technology, 5600 MB Eindhoven, The Netherlands
}

(Received 30 March 2015; published 14 September 2015)

\begin{abstract}
We report the realization of quantum microwave circuits using hybrid superconductor-semiconductor Josephson elements comprised of InAs nanowires contacted by NbTiN. Capacitively shunted single elements behave as transmon circuits with electrically tunable transition frequencies. Two-element circuits also exhibit transmonlike behavior near zero applied flux but behave as flux qubits at half the flux quantum, where nonsinusoidal current-phase relations in the elements produce a double-well Josephson potential. These hybrid Josephson elements are promising for applications requiring microwave superconducting circuits operating in a magnetic field.
\end{abstract}

DOI: 10.1103/PhysRevLett.115.127002

PACS numbers: 85.25.Hv, 62.23.Hj, 74.45.+c, 84.40.Dc

In superconducting circuits, macroscopic degrees of freedom like currents and voltages can exhibit quantum mechanical behavior. These circuits can behave as artificial atoms with discrete, anharmonic levels whose transitions can be driven coherently [1]. In the field of circuit quantum electrodynamics (cQED), these artificial atoms are coupled to resonators to perform microwave quantum optics in the solid state [2,3]. Over the past decade, cQED has also grown into a promising platform for quantum information processing, wherein the ground and first-excited levels of each atom serve as an effective qubit [4]. To date, implementations of superconducting quantum circuits have relied almost exclusively on aluminum-aluminum-oxidealuminum $(\mathrm{Al} / \mathrm{AlOx} / \mathrm{Al})$ tunnel junctions as the source of nonlinearity without dissipation. However, many exciting applications require magnetic fields $(\sim 0.5 \mathrm{~T})$ at which superconductivity in aluminum is destroyed, calling for an alternative approach to realizing microwave artificial atoms.

Recent advances in materials development and nanowire (NW) growth have enabled the development of superconductor-semiconductor structures supporting coherent charge transport without dissipation [5] and providing signatures of Majorana bound states [6]. To date, superconductor-semiconductor-superconductor Josephson elements (JEs) have been studied exclusively in quasi-dc [7-10] and radio-frequency [11] transport. Building microwave circuits operating in the quantum regime, in which transition energies between levels exceed the thermal energy, offers new ways to investigate the physics of hybrid superconductor-semiconductor structures using spectroscopy [12-15].

In this Letter, we report the realization of microwavefrequency $\mathrm{CQED}$ circuits made from hybrid JEs based on
InAs NWs contacted by NbTiN. Capacitively shunted single JEs behave as weakly anharmonic oscillators, or transmons [16], with transition frequencies tunable by the field effect, i.e., voltage on a proximal side gate. Doubleelement devices show similar transmonlike behavior at zero applied flux but behave as flux qubits [17] near full frustration owing to a double-well Josephson potential arising from nonsinusoidal current-phase relations (CPR s). We observe microwave-driven transitions between states with oppositely flowing persistent currents, manifesting macroscopic quantum coherence. Their fabrication from magnetic-field-compatible materials makes these JEs promising for applications requiring quantum microwave circuits withstanding magnetic fields up to $0.5 \mathrm{~T}$, such as the braiding of Majorana particles and the interfacing with coherent quantum memories based on polarized electronspin ensembles.

Our chip [Fig. 1(a)] contains multiple capacitively shunted single and double NW JEs coupled to dedicated transmission-line resonators for control and readout using a common feed line [Fig. 1(b)]. The chip contains side gates for electrostatic tuning of some single-junction devices and current-bias lines for threading flux through the loops of split-junction devices. We create each JE by deterministically placing an InAs NW between the leads of a prepatterned NbTiN interdigitated capacitor and contacting the NW to each lead in a subsequent NbTiN deposition. The charging energy $E_{C} \approx h \times 300 \mathrm{MHz}$ of the devices is chosen much smaller than the estimated Josephson coupling energy $E_{J}$ of the NW junction, as in conventional transmon devices [16], leading to a weakly anharmonic energy spectrum (energies $E_{i}$ ) of circuit plasma modes. We first verify the presence of the nonlinear NW circuit by measuring the feed line transmission near the fundamental 


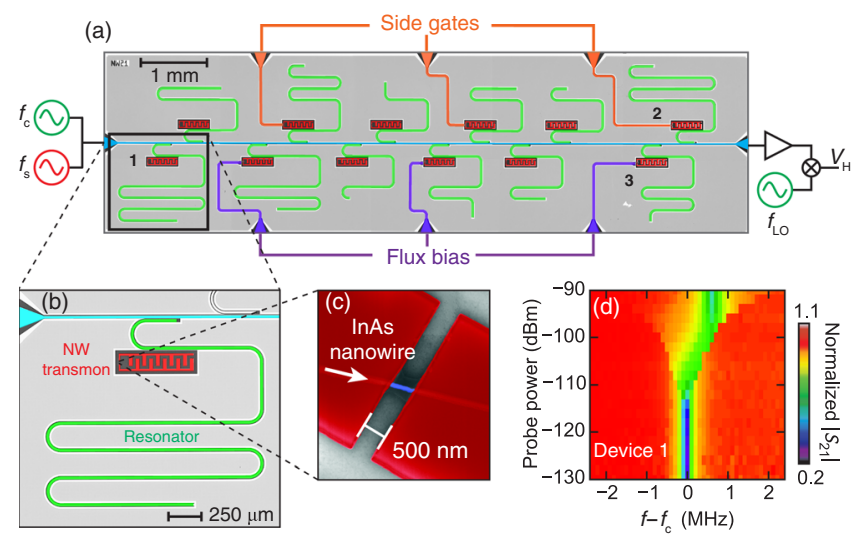

FIG. 1 (color online). Hybrid Josephson elements in cQED. (a) Overview of a cQED chip allowing control and readout of NW circuits using dedicated resonators (green) coupled to a common feed line (blue). For readout, a microwave tone with frequency $f_{c}$ is applied near the fundamental of the resonator coupling to the NW circuit under study. The signal is amplified and downconverted to $1 \mathrm{MHz}$ using a local oscillator at $f_{\mathrm{LO}}$ for subsequent digitization and processing. Additional controls on a subset of devices include side gates (orange) for electrostatic tuning of carrier density in the NW of single-junction devices and shortcircuited transmission lines (purple) for threading flux through the loops of split-junction devices. A circuit diagram detailing the readout and control circuitry is shown in Fig. S2 of Supplemental Material [21]. Measurements on devices 1-3 are described in the main text. See Table S1 in Supplemental Material [21] for an overview of the other devices. (b) Optical enlargement of device 1, containing a single-junction NW circuit (red). (c) Scanning electron microscope (SEM) image of an InAs NW (blue) contacted by NbTiN electrodes (red) separated by $500 \mathrm{~nm}$. (d) Normalized feed line transmission as a function of readout power. The resonator shifts from $f_{c}=3.9464 \mathrm{GHz}$ at singlephoton level to $f_{\text {bare }}=3.9470 \mathrm{GHz}$ above $10^{5}$ photons. This shift confirms the coupling of the resonator to a nonlinear circuit.

frequency of the coupled resonator [Fig. 1(d)]. The JaynesCummings interaction leads to different resonator frequencies $f_{c}$ and $f_{\text {bare }}$ at single- and many-photon probe levels, respectively $[18,19]$. We then search for the qubit transition frequency $f_{01}$ of the NW circuit by monitoring feed line transmission at $f_{c}$ while sweeping a second tone [20] near the estimated frequency $f_{\text {bare }}+g^{2} /\left(f_{\text {bare }}-f_{c}\right)$, where $g$ is the coupling strength between the NW circuit and resonator.

We first investigate the electric-field effect on the NWcircuit spectrum [Fig. 2(a)]. Device 2 has one NW junction (measured length $L \approx 550 \mathrm{~nm}$ ) and a proximal side-gate electrode for tuning the carrier density in the NW. Transmons have exponentially suppressed charge dispersion when $E_{J} \gg E_{C}$. Side-gate tuning therefore influences the excitation spectrum of the circuit only through the electric-field effect on the NW. We observe fully reproducible fluctuations [21] in $f_{01}$ as a function of the side-gate voltage $V_{g}$, indicating phase-coherent diffusive charge transport in the NW $[5,29]$. Using the plasma-oscillation relation [16]
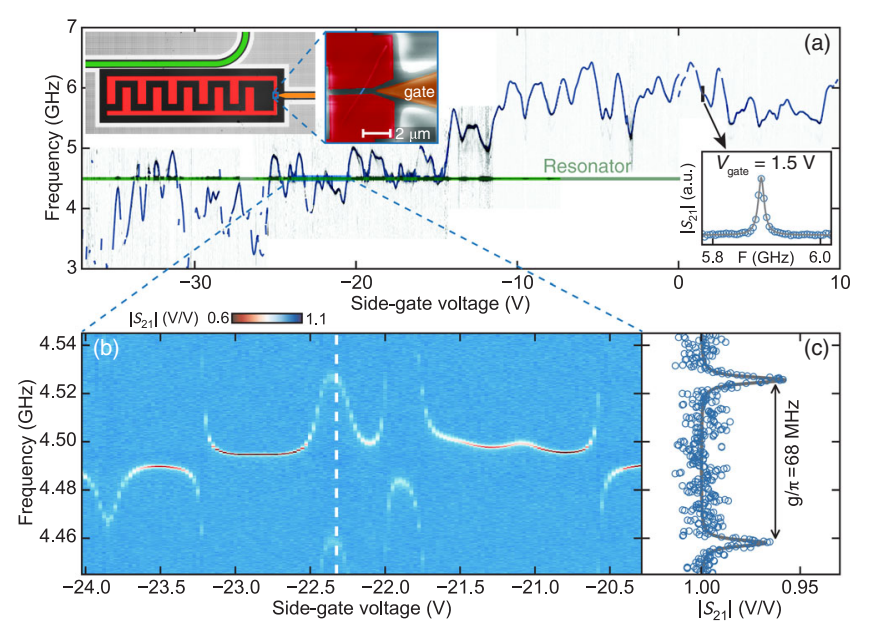

FIG. 2 (color online). Side-gate tuning of a NW circuit and mesoscopic Josephson coupling fluctuations. (a) Left inset: False-colored optical image of device 2. Right inset: SEM micrograph showing the single $\mathrm{NW}$ junction and the proximal side gate (orange) for voltage control. Sweeping this voltage induces reproducible fluctuations in the fundamental transition frequency $f_{01}$. Lower inset: Example spectroscopy of the fundamental transition, showing an inhomogeneously broadened linewidth $\gamma / 2 \pi=13.2 \pm 0.3 \mathrm{MHz}$. A downward trend in $f_{01}$ is observed as $V_{g}$ decreases. At $V_{g}<-15 \mathrm{~V}, f_{01}$ fluctuates around the resonator fundamental (green line). (b) An enlargement around $V_{g}=-22 \mathrm{~V}$ shows multiple avoided crossings. (c) At $V_{g}=-22.3 \mathrm{~V}$, the NW circuit fully hybridizes with the resonator. From the minimum splitting, we extract the NW circuitresonator coupling strength $g / 2 \pi=34 \pm 1 \mathrm{MHz}$.

$E_{J} \approx f_{01}^{2} / 8 E_{C}$, we determine the root-mean-square Josephson-energy fluctuation $\sqrt{\left\langle\delta^{2} E_{J}\right\rangle} / h \approx 2 \mathrm{GHz}$ in the $V_{g}=0-10 \mathrm{~V}$ range. Matching this scale to the Thouless energy [30] $E_{\mathrm{Th}}=\hbar D / L^{2}$ and assuming highly transparent contacts [9], we estimate the diffusion constant $D \approx 40 \mathrm{~cm}^{2} / \mathrm{s}$. This value is typical for InAs wires [7].

Side-gate tuning of the NW junction offers a new means to control the spectrum of transmons. Decreasing $V_{g}$ brings $f_{01}$ into resonance with the resonator, revealing multiple avoided crossings [Fig. 2(b)]. The minimum splitting indicates $g / 2 \pi=34 \pm 1 \mathrm{MHz}$ [Fig. 2(c)]. We note that while we perform only quasistatic field-effect tuning of $f_{01}$ throughout this experiment, nanosecond control should be possible by increasing the bandwidth of off-chip filtering.

We now discuss the impact of charge fluctuations on the $f_{01}$ transition and the observed minimum linewidth, which will be of interest for qubit applications. Field-effect tuning of the Josephson coupling can make $f_{01}$ sensitive to nearby fluctuating charges. One may expect a region with $\partial f_{01} / \partial V_{g}=0$ to constitute a charge sweet spot [31] and thus to correlate with a linewidth reduction. However, we do not observe a correlation between the linewidth $\gamma / 2 \pi>$ $10 \mathrm{MHz}$ and $\left|\partial f_{01} / \partial V_{g}\right|$, suggesting a different dominant decoherence channel [21]. We surmise a connection between this broad linewidth and the soft gap induced in 


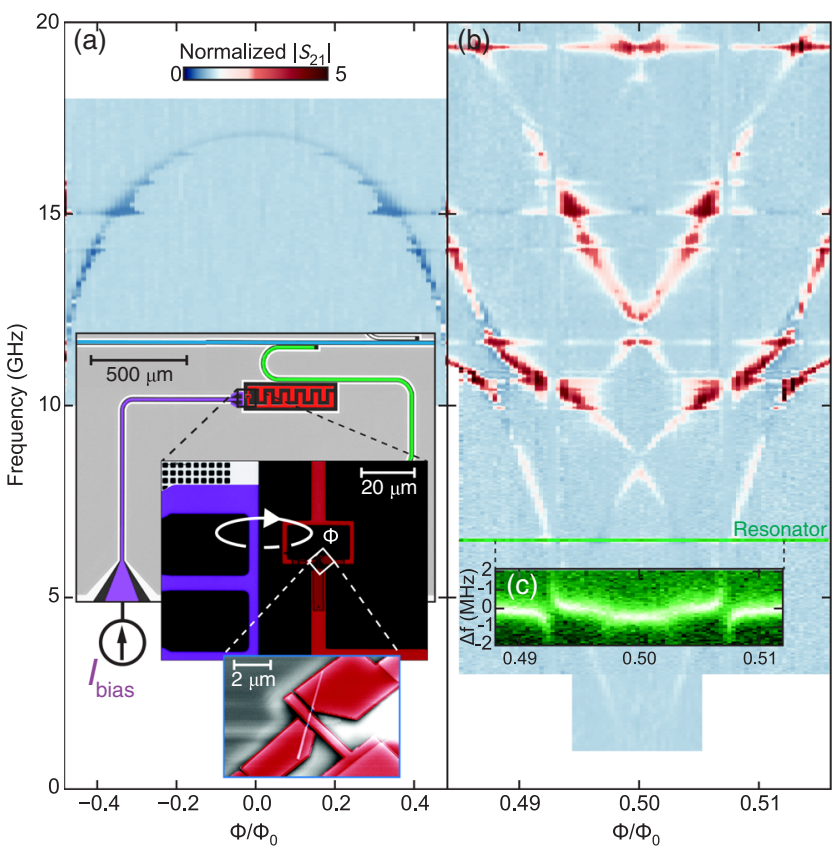

FIG. 3 (color online). Flux-bias spectroscopy of a split-junction NW circuit. (a) Inset: False-colored optical image showing device 3 (red), its resonator (green), and flux-bias line (purple). Bottom inset: SEM micrograph of the two JEs made from one NW. Flux-bias spectroscopy shows the tuning of $f_{01}$ with $\Phi$. (b) A high-resolution sweep around $\Phi=\Phi_{0} / 2$ shows a strong flux dependence of the NW circuit transitions. (c) Measurement of resonator transmission around $f_{c}$ with the same horizontal range as in (b). The avoided crossing of the lowest transition with the resonator reveals a much reduced coupling strength.

NWs [32] contacted by NbTiN using similar fabrication techniques [6]. Parallel experiments by the Copenhagen group achieve hard induced gaps in epitaxial Al-InAs NWs [33] and $\sim 1 \mu$ s coherence times in a single-JE hybrid transmon [34]. Establishing a clear connection between a soft induced gap and dissipation in microwave circuits will be the focus of future studies.

Next, we consider a split-junction device where the two parallel JEs (each with $L=150 \mathrm{~nm}$ ) are created from one $5 \mu \mathrm{m}$ long NW [Fig. 3(a)]. As in conventional transmons, $f_{01}$ first decreases as flux $\Phi$ is threaded through the loop. However, near $\Phi \sim \Phi_{0} / 2$ ( $\Phi_{0}=h / 2 e$ is the flux quantum), a clear departure from transmonlike behavior is observed [Fig. 3(b)]. Multiple strongly flux-dependent transitions and a new, strong avoided crossing appear symmetrically about $\Phi_{0} / 2$. In addition, the avoided crossing between the lowest transition and the resonator is strongly reduced [Fig. 3(c)] compared to that of device 2 [35]. The highly anharmonic spectrum and much reduced coupling to the resonator show that the states associated with the lowestenergy transition are no longer plasma modes.

The observed deviation from the conventional transmon energy spectrum provides a signature of nonsinusoidal CPR $\mathrm{s}$ in the NW junctions [36]. We now show that the observed spectrum can be fully explained by the Hamiltonian of a Cooper-pair box: $H=4 E_{C} \hat{N}+V_{1}(\hat{\delta})+$ $V_{2}\left(2 \pi \Phi / \Phi_{0}-\hat{\delta}\right)$, provided its split junctions do not follow a cosine-shaped Josephson potential [Fig. 4(a)]. Here, the operators $\hat{N}$ and $\hat{\delta}$ represent the charge imbalance between islands and the phase difference across NW junction 1, respectively. The Josephson potential $V_{i}\left(\varphi_{i}\right)$ of junction $i$ is linked to its CPR by $I_{i}\left(\varphi_{i}\right)=\left(2 \pi / \Phi_{0}\right) \partial V_{i} / \partial \varphi_{i}$, where $\varphi_{1} \equiv \hat{\delta}$ and $\varphi_{2} \equiv 2 \pi \Phi / \Phi_{0}-\hat{\delta}$. Crucially, we require $V_{i}$ to be $2 \pi$-periodic but not necessarily cosine shaped. Using a simple phenomenological model $[21,30]$ of the form $V_{i}\left(\varphi_{i}\right)=-K_{i} \sqrt{1-T_{i} \sin ^{2}\left(\varphi_{i} / 2\right)}$ and performing a nonlinear least-squares fit with five free parameters, we obtain a quantitative match to all spectral data (best-fit values are $E_{C} / h=279 \pm 1 \mathrm{MHz}, K_{1} / h=376 \pm 13 \mathrm{GHz}, K_{2} / h=$ $233 \pm 2 \mathrm{GHz}, T_{1}=0.86 \pm 0.02$, and $T_{2}=0.885 \pm 0.004$ ). This simple model strictly applies only to short weak links having a single conductance channel; we therefore use the

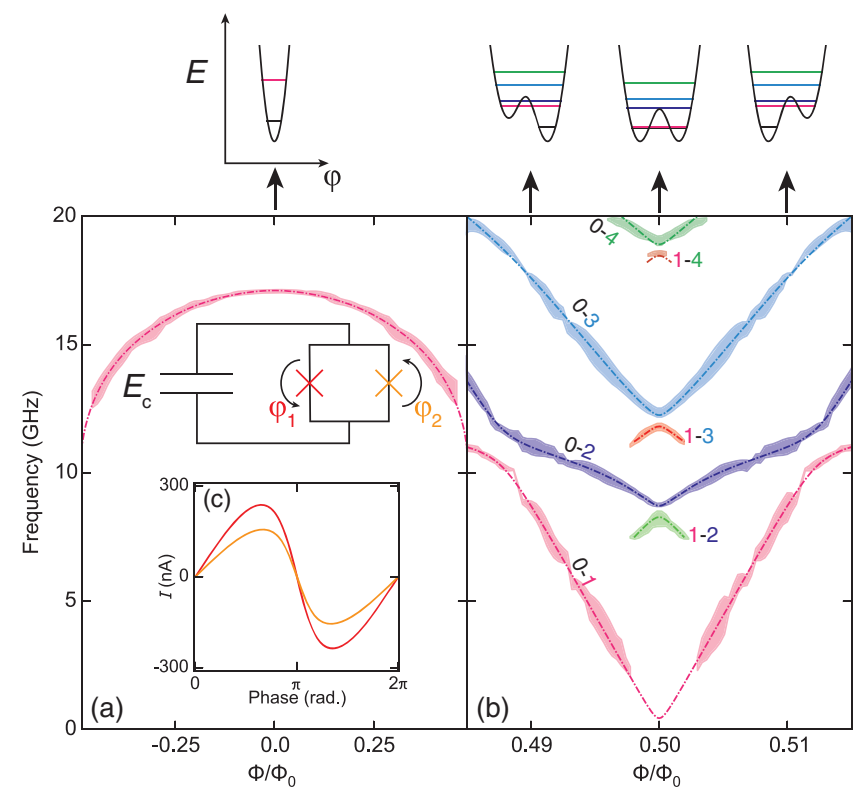

FIG. 4 (color online). Fitting theory to the spectrum of the split-junction NW circuit. (a) Top inset: Equivalent circuit of the device, a split Cooper-pair box containing a loop interrupted by two NW JEs (phase differences $\varphi_{1,2}$ ), threaded by an externally applied flux $\Phi$ and shunted by a capacitance, giving total charging energy $E_{C}$. Around $\Phi=0$, the Josephson potential has a single minimum, producing a weakly anharmonic spectrum. (b) The nonsinusoidal current-phase relation of the NW JEs determines the particular flux dependence of the transition frequencies around $\Phi=\Phi_{0} / 2$ as the Josephson potential develops a symmetric double-well profile that tilts as $\Phi$ is tuned away from $\Phi_{0} / 2$. All curves are the result of a least-squares nonlinear fit of the theoretical model described in the text. We identify four fundamental transitions from the ground state and three transitions from the first-excited state. (c) The CPR calculated from the fit of the spectrum to the theoretical model described in the text. 
fit only to extract information about the CPR $\mathrm{s}$ of the junctions. As shown in Fig. 4(c), the corresponding CPR s are evidently skewed. A three-parameter fit using $V_{i}$ corresponding to the CPR of a chaotic junction in the many-channel limit [24] shows only slightly worse agreement, as does a truncated Fourier series expansion of $V_{i}$. All approaches produce similar skewed CPR s [21].

Interestingly, this device can be operated in two distinct regimes by tuning $\Phi$. Near $\Phi=0$, it operates like a transmon, whose eigenstates are plasma modes with a weakly anharmonic spectrum. Around $\Phi \simeq \Phi_{0} / 2$, it operates like a flux qubit [17] whose two lowest energy levels carry opposite persistent currents $I_{p, i}=\partial E_{i} / \partial \Phi$, which we estimate to be of the order of $\pm 100 \mathrm{nA}$ [21]. The possibility to drive transitions between these distinct persistent-current states using coherent microwaves constitutes a manifestation of macroscopic quantum coherence [37] in our NW circuits.

In conclusion, we have realized hybrid microwave circuits made from superconductor-semiconductor NW JEs and characterized them using spectroscopy. NW circuits offer several advantages over traditional aluminum circuits. First, tuning transitions using the field effect in single NW JE devices offers an alternative to flux biasing of split-junction $\mathrm{Al}$ devices, with the potential to reduce on-chip dissipation by eliminating the need for mA-level currents. Second, the hybrid JEs are made exclusively from magnetic-field-compatible materials [38,39]. Magneticfield-compatible superconductor-semiconductor NW microwave circuits will open new avenues of research. In particular, very pure solid-state electron spin ensembles (e.g., nitrogen impurities in diamond or phosphorous donors in silicon) could be field polarized to make coherent quantum memories $[39,40]$ for hybrid quantum processors. In addition, hybrid microwave quantum circuits and the cQED architecture offer a way to study mesoscopic superconductivity and Andreev bound states [28] in NW JEs and to read out and control Majorana bound states [41,42] in proposed demonstrations of non-Abelian exchange statistics [43-46]. Apart from improving quantum coherence, immediate next experiments will therefore focus on the study of these circuits in up to $\sim 0.5 \mathrm{~T}$ in-plane magnetic fields.

We thank L.P. Kouwenhoven, T. M. Klapwijk, M. H. Devoret, C. W. J. Beenakker, and J. E. Mooij for useful discussions and comments on the manuscript. We acknowledge funding by Microsoft Corporation Station Q, the Dutch Organization for Fundamental Research on Matter (FOM), the Netherlands Organization for Scientific Research (NWO), and an ERC Synergy Grant.

[1] J. Clarke and F. K. Wilhelm, Nature (London) 453, 1031 (2008).

[2] A. Blais, R.-S. Huang, A. Wallraff, S. M. Girvin, and R. J. Schoelkopf, Phys. Rev. A 69, 062320 (2004).
[3] A. Wallraff, D. I. Schuster, A. Blais, L. Frunzio, R.-S. Huang, J. Majer, S. Kumar, S. M. Girvin, and R. J. Schoelkopf, Nature (London) 431, 162 (2004).

[4] M. H. Devoret and R. J. Schoelkopf, Science 339, 1169 (2013).

[5] Y.-J. Doh, J. A. v. Dam, A. L. Roest, E. P. A. M. Bakkers, L. P. Kouwenhoven, and S. D. Franceschi, Science 309, 272 (2005).

[6] V. Mourik, K. Zuo, S. M. Frolov, S. R. Plissard, E. P. A. M. Bakkers, and L.P. Kouwenhoven, Science 336, 1003 (2012).

[7] T. S. Jespersen, M. L. Polianski, C. B. Sørensen, K. Flensberg, and J. Nygård, New J. Phys. 11, 113025 (2009).

[8] N. L. B. Ziino, P. Krogstrup, M. H. Madsen, E. Johnson, J. B. Wagner, C. M. Marcus, J. Nygård, and T. S. Jespersen, arXiv:1309.4569.

[9] S. Abay, D. Persson, H. Nilsson, F. Wu, H. Q. Xu, M. Fogelström, V. Shumeiko, and P. Delsing, Phys. Rev. B 89, 214508 (2014).

[10] I. Sochnikov et al., Phys. Rev. Lett. 114, 066801 (2015).

[11] M. Ebel, C. Busch, U. Merkt, M. Grajcar, T. Plecenik, and E. Il'ichev, Phys. Rev. B 71, 052506 (2005).

[12] D. Pekker, C.-Y. Hou, V. E. Manucharyan, and E. Demler, Phys. Rev. Lett. 111, 107007 (2013).

[13] C. Müller, J. Bourassa, and A. Blais, Phys. Rev. B 88, 235401 (2013).

[14] E. Ginossar and E. Grosfeld, Nat. Commun. 5, 4772 (2014).

[15] J. I. Väyrynen, G. Rastelli, W. Belzig, and L. I. Glazman, arXiv:1504.03680.

[16] J. Koch, T. M. Yu, J. Gambetta, A. A. Houck, D. I. Schuster, J. Majer, A. Blais, M. H. Devoret, S. M. Girvin, and R. J. Schoelkopf, Phys. Rev. A 76, 042319 (2007).

[17] J. E. Mooij, T. P. Orlando, L. Levitov, L. Tian, C. H. van der Wal, and S. Lloyd, Science 285, 1036 (1999).

[18] M. D. Reed, L. DiCarlo, B. R. Johnson, L. Sun, D. I. Schuster, L. Frunzio, and R. J. Schoelkopf, Phys. Rev. Lett. 105, 173601 (2010).

[19] L. S. Bishop, E. Ginossar, and S. M. Girvin, Phys. Rev. Lett. 105, 100505 (2010).

[20] J. A. Schreier et al., Phys. Rev. B 77, 180502 (2008).

[21] See Supplemental Material at http://link.aps.org/ supplemental/10.1103/PhysRevLett.115.127002 for device details, experimental methods and extended data analysis, which includes Refs. [22-28].

[22] M. Scheffler, S. Nadj-Perge, L. P. Kouwenhoven, M. T. Borgström, and E. P. A. M. Bakkers, Physica (Amsterdam) E 40, 1202 (2008).

[23] A dynamic IPython Notebook containing the relevant code is available at http://goo.gl/3xfGr8.

[24] P. W. Brouwer and C. W. J. Beenakker, Chaos Solitons Fractals 8, 1249 (1997).

[25] H. U. Baranger and P. A. Mello, Phys. Rev. Lett. 73, 142 (1994).

[26] R. Jalabert, J.-L. Pichard, and C. Beenakker, Europhys. Lett. 27, 255 (1994).

[27] V. E. Manucharyan, J. Koch, L. I. Glazman, and M. H. Devoret, Science 326, 113 (2009).

[28] L. Bretheau, Ç. Ö. Girit, H. Pothier, D. Esteve, and C. Urbina, Nature (London) 499, 312 (2013).

[29] P. A. Lee and A. D. Stone, Phys. Rev. Lett. 55, 1622 (1985). 
[30] C. W. J. Beenakker, Phys. Rev. Lett. 67, 3836 (1991).

[31] D. Vion, A. Aassime, A. Cottet, P. Joyez, H. Pothier, C. Urbina, D. Esteve, and M. H. Devoret, Science 296, 886 (2002).

[32] S. Takei, B. M. Fregoso, H.-Y. Hui, A. M. Lobos, and S. Das Sarma, Phys. Rev. Lett. 110, 186803 (2013).

[33] W. Chang, S. M. Albrecht, T. S. Jespersen, F. Kuemmeth, P. Krogstrup, J. Nygård, and C. M. Marcus, Nat. Nanotechnol. 10, 232 (2015).

[34] T. W. Larsen, K. D. Petersson, F. Kuemmeth, T. S. Jespersen, P. Krogstrup, J. Nygård, and C. M. Marcus, preceding Letter, Phys. Rev. Lett. 115, 127001 (2015).

[35] In addition, several flux-independent lines appear. We can attribute many of these to the fundamentals and higher harmonics of other resonators on the chip (they are also observed when studying other devices on this chip) and are not considered henceforth.

[36] A. A. Golubov, M. Y. Kupriyanov, and E. Il'ichev, Rev. Mod. Phys. 76, 411 (2004).
[37] A. J. Leggett, Prog. Theor. Phys. Suppl. 69, 80 (1980).

[38] L. P. Rokhinson, X. Liu, and J. K. Furdyna, Nat. Phys. 8, 795 (2012).

[39] V. Ranjan, G. de Lange, R. Schutjens, T. Debelhoir, J. P. Groen, D. Szombati, D. J. Thoen, T. M. Klapwijk, R. Hanson, and L. DiCarlo, Phys. Rev. Lett. 110, 067004 (2013).

[40] A. Imamoglu, Phys. Rev. Lett. 102, 083602 (2009).

[41] J. Alicea, Y. Oreg, G. Refael, F. von Oppen, and M. P. Fisher, Nat. Phys. 7, 412 (2011).

[42] T. Hyart, B. van Heck, I. C. Fulga, M. Burrello, A. R. Akhmerov, and C. W. J. Beenakker, Phys. Rev. B 88, 035121 (2013).

[43] G. Moore and N. Read, Nucl. Phys. B360, 362 (1991).

[44] N. Read and D. Green, Phys. Rev. B 61, 10267 (2000).

[45] D. A. Ivanov, Phys. Rev. Lett. 86, 268 (2001).

[46] C. Nayak, S. H. Simon, A. Stern, M. Freedman, and S. D. Sarma, Rev. Mod. Phys. 80, 1083 (2008). 